Research Article

\title{
Customized Preventive Maintenance Strategies for Products Sold with Two-Dimensional Warranty
}

\author{
Fangqi Dong $\left(\mathbb{D},{ }^{1}\right.$ Huaqiang Wang $\mathbb{D},{ }^{2}$ Feng Men $\mathbb{D},{ }^{1}$ and Lijun Liang ${ }^{3}{ }^{3}$ \\ ${ }^{1}$ China Automotive Technology and Research Center Co., Ltd., Tianjin 300300, China \\ ${ }^{2}$ School of Sports Economics and Management, Tianjin University of Sport, Tianjin 301617, China \\ ${ }^{3}$ College of Management, Tianjin University of Traditional Chinese Medicine, Tianjin 300073, China
}

Correspondence should be addressed to Lijun Liang; lianglijun1@163.com

Received 13 January 2021; Revised 22 February 2021; Accepted 12 March 2021; Published 27 March 2021

Academic Editor: Zeljko Stevic

Copyright ( $\odot 2021$ Fangqi Dong et al. This is an open access article distributed under the Creative Commons Attribution License, which permits unrestricted use, distribution, and reproduction in any medium, provided the original work is properly cited.

Two-dimensional warranty has played a significant role in improving product quality and competitiveness. However, twodimensional basic warranty (BW) and extended warranty (EW) have not yet been combined effectively, and the customized maintenance strategies based on the consumers' heterogeneity have been quite limited, which result in high maintenance costs. In this paper, the possibility of reducing warranty cost over BW and EW is explored by classifying customers based on their usage rates and then offering them with customized preventive maintenance (PM) strategies. By considering the purchasing ratio of EW contracts, the PM strategies for BW and EW are closely combined by the bi-level programming model. Numerical analysis is given to show the application of the proposed warranty strategy, and the significant findings and sensitivity of the main parameters are analyzed in the end. The findings demonstrate that the customized PM strategies combining BW and EW can effectively reduce warranty costs within the warranty period and provide a scientific guidance for manufacturers to design a more reasonable PM strategy.

\section{Introduction}

In an increasingly fierce competitive market environment, many manufacturers begin to offer attractive warranty service for capturing more market share and customer loyalty. A warranty is a contractual agreement between the customer and the manufacturer, which provides a detailed description of the manufacturer's responsibilities in the event that the product cannot operate satisfactorily when used correctly [1]. According to the number of parameters defining warranty coverage, warranty policies can be generally classified into one-dimensional warranty and twodimensional warranty $[2,3]$. One-dimensional warranty is often characterized by age limit or usage limit, while twodimensional warranty considers both age limit and usage limit or the internal relation between them. Actually, the product failures of consumer durable goods like engineering machinery and autos are indexed by both age and usage. Moreover, in order to attract consumers and improve competitive advantages, manufacturers often make an effective two-dimensional warranty policy, in which the product's age and usage are considered simultaneously. Generally, two-dimensional warranties can be extended to apply in the durable goods mentioned above. For instance, a car is generally sold with a two-dimensional warranty, providing free repair for $60000 \mathrm{~km}$ or 3 years, whichever comes up first.

Within the warranty coverage, corrective maintenance $(\mathrm{CM})$ and preventive maintenance (PM) are usually implemented for warranty service [4]. A CM action is generally unscheduled to bring a failed product back to its operating status, and it would be minimal, which restores the failed product to the original state, or imperfect, which repairs the failed product back to a status between as good as the original state and as same as failed. In addition, a CM action could be perfect as a replacement by bringing the product to a state akin to the original version [4]. Compared with a CM action, a PM action, the level of which can be decided and governed by the manufacturers, is planned action either to lower the failure probability or to lengthen 
the lifetime while the product is still in the operational status, which can also be either perfect or imperfect [2]. At the price of additional PM costs, the warranty cost can be effectively decreased by performing PM actions, which can be enforced by slowing down the degradation process of the product, when the decrement in the warranty cost surpasses the expense of PM activities [5].

Usually, manufacturers offer a free BW service for those customers buying a new product. In product sales, customers are often offered an option of purchasing an EW contract that offers protection for an extended period after the BW period expires, which can offer considerable profit margins for manufacturers and contribute to keep in contact with customers beyond the period of BW [6]. Due to the growing complexity of consumer durable goods in both functions and structures, the repair of such products is more sophisticated and expensive. Therefore, a reasonable maintenance policy has a remarkable influence on reducing the warranty cost covered by the manufacturer [2]. In recent years, how to apply the right PM strategies for products covered by two-dimensional BW or EW contract is a great challenge for the manufacturers, which has attracted significant attention among practitioners and academics [7-10].

The related work of PM strategies for two-dimensional warranty can be grouped into three main types based on warranty coverage, such as BW, EW, and both BW and EW [11-15]. For products covered by BW, Wang and Su [10] prove that compared to periodic PM programs, two-dimensional PM based on both the age and usage of products can effectively reduce the warranty servicing cost. For a dump truck sold with different two-dimensional BW contracts, Nasrum et al. [13] suggest that preventive maintenance with different time intervals can effectively reduce warranty servicing cost, which is examined by a numerical example. Considering both product availability and warranty cost, a warranty service combining minimal repair and the imperfect preventive maintenance is presented by Cheng et al. [5] to minimize the product's cost-efficiency per unit time. As is well known, the maintenance strategies for products under BW have an important influence on the maintenance followed by EW. Under the assumption that minimal repair is executed when the product failed during the BW, the optimal PM strategies for two-dimensional EW have been studied by Shahanaghi [14], for which the optimal PM time interval and maintenance degree are obtained by computational analysis.

Most of the abovementioned researches mainly focus on the optimization of schedule intervals or the maintenance degree of the unitary PM strategy. However, due to the heterogeneity of customers' usage rates or risk preference, the unitary PM strategy cannot meet the different demands of all the customers. Thus, the concept of customized PM strategy is proposed by SU [16], who suggests that the PM strategies with different time intervals or maintenance degrees can be provided to the customers over the EW coverage according to their usage rates, which can significantly lower the maintenance cost as a whole. Then, Huang et al. [17] propose a customized PM strategy for products during the EW period analogously, in which customers are divided into three groups on the basis of their maintenance records during the BW period, and customers in the different groups are offered different PM schedules. The results show that providing customers with customized PM can not only lower the warranty costs but also be a tangible competitive strategy for manufacturers. For all we know, the customized PM programs with two-dimensional warranty are quite limited, which cannot meet the demand of practitioners and academics.

The above researches for the optimal PM strategies have not incorporated BW and EW as a whole; therefore, it is practically impossible to get optimal warranty under either BW or EW coverage, when considering the whole warranty service. In the manufacturer's view, it is absolutely essential to combine the BW and EW contracts, and calculate the entire expected warranty cost to the manufacturer, for the purpose of deriving the optimal PM strategy within the whole warranty period $[2,16]$. By applying different PM strategies for products over the BW and EW periods, Bouguerra et al. [18] obtained the highest two-dimensional EW cost acceptable to the consumers, and the lowest twodimensional EW price acceptable to the manufacturers, which provide convenience for pricing of the two-dimensional EW service. Subsequently, BW and EW are combined into a whole warranty period by Wang et al. [2], and the optimal PM strategy under the whole two-dimensional warranty is obtained, for which the PM intervals and maintenance degrees during BW and EW are different, and the total warranty cost is the minimum.

In the researches mentioned above, the maintenance strategies combining the BW and EW take no account of the EW purchasing ratio, which may have a significant influence on the maintenance strategies for both BW and EW, since not everyone will purchase the EW contracts when the BW period expires. Furthermore, customers in the market are different in usage rates, which would have a major impact on the optimization of maintenance strategies $[17,19]$. Motivated by this, the study designs a customized periodic PM strategy for the customers with different usage rates, and then combines the maintenance strategies between BW and EW with the bi-level programming model, for which the warranty servicing cost of the manufacturers is minimum as a whole.

The remaining study is arranged as follows. In Section 2, the modeling assumption and notations used in this paper are described. In Section 3, the model analysis is presented to estimate the expected warranty cost under the periodic and imperfect PM schedule. In Section 4, the optimal PM strategies model that combines BW and EW is created based on the bi-level programming model. In Section 5, an illustrative numerical example is adopted to evaluate the performance of the proposed strategy and the sensitivity analysis of the main parameters. Finally, the major conclusions and some topics for future research are discussed in Section 6.

\section{Model Assumptions and Notations}

The model assumptions and main mathematical notations used in this study are given in this section. 


\subsection{Model Assumptions}

(1) For a given customer, the usage rate over the $\mathrm{BW}$ and EW is constant, but it varies from customer to customer. The distribution of usage rates for all customers is obtained by summarizing the sales records of products from the dealers or surveying the customers.

(2) When the BW period expires, the customer can purchase an EW contract at a fixed price. All the product failures are statistically independent, and the manufacturer is responsible for minimally repairing and bearing the total maintenance cost during the warranty coverage.

(3) The periodic PM is designed and performed to slow down the degradation process of the product during the whole warranty period, and it is assumed that the level of preventive repair is adjustable.

(4) The time interval and maintenance degree of the PM can be different between BW and EW but must be the same for the same warranty period. The PM cost during the whole warranty period is undertaken by the manufacturer. Compared to the time to product failure, the time to minimal repair and PM is very short and negligible.

2.2. Model Notations. The mathematical notations and descriptions applied in the study are given in Table 1 .

\section{Product Failure and Periodic PM Strategy Model}

3.1. Product Failure Model. At present, marginal (univariate) method, bivariate failures distribution method, and composite scale method have been proposed for modeling product failures under two-dimensional warranty [20-22]. In this paper, the marginal method is used to model the process of product failure. Set $x$ and $u$ as the product's age and usage, respectively, and $(t, u)=(0,0)$ corresponds to the point of product purchase. In the marginal method, the usage rate $R$ is assumed as a random variable varying with the customer population; however, a specific customer's usage rate $R$ is constant over time. Hence, distribution function $G(r)=P(R<r)$ and cumulative distribution function $g(r)$ can be adopted to model $R$. Conational on $R=r$, the product's overall usage $u$ at age $t$ can be calculated by $u=r t$.

Assuming that manufacturers minimally repair all failures with negligible duration and do not implement any PM strategy, the failures over time follow a nonhomogeneous Poisson process (NHPP), in which the conditional failure intensity function is $\lambda(t \mid r)=\varphi(t, u)$, where $\varphi(t, u)$ denotes nondecreasing function of both $t$ and $u$. Therefore, a point process with an intensity function can be adopted to model product failure, which is dependent on both $t$ and $u$. As described by Murthy et al. [23], this paper models the conditional intensity function as a polynomial function, which can be expressed as

$$
\lambda_{0}(t \mid r)=\theta_{0}+\theta_{1} r+\left(\theta_{2}+\theta_{3} r\right) t, \quad \theta_{0}, \theta_{1}, \theta_{2}, \theta_{3}>0
$$

where $\theta_{0}, \theta_{1}, \theta_{2}, \theta_{3}$, which are positive constants, can be calculated through the manufacturer's database of warranty claims and maintenance records. The two-dimensional failure model can be simplified to a one-dimensional model by using the marginal approach. It is noteworthy that the two-dimensional warranty usually uses the polynomial intensity function in the literature $[2,8,15,16,24]$ for reference.

As can be seen, customer usage rate $r$ is a critical factor influencing the degradation process of the product, so as to optimize the maintenance strategy for the product during the whole warranty period. In this paper, the customers are divided into different categories based on their usage rates; then, customized PM strategy can be provided for customers in the different categories. For the given $G(r)$ and $g(r)$, set $r_{\min }, r_{\max }$ as the minimum usage rate and maximum usage rate, respectively, then the $i^{\text {th }}$ usage rate interval $\left[r_{i-1}, r_{i}\right), i=$ $1, \ldots, N$ can be calculated by

$$
\begin{aligned}
& r_{0}=r_{\text {min }}, \\
& r_{i}=r_{\min }+i * \frac{r_{\text {max }}-r_{\text {min }}}{N}, \quad i=1,2, \ldots, N,
\end{aligned}
$$

where $N$ denotes the number of usage rate scenarios; for any given customer, the probability of his usage rate $r$ dropping into the $i^{\text {th }}$ usage rate interval is

$$
P_{r i}=G\left(r_{i}\right)-G\left(r_{i-1}\right), \quad i=1, \ldots, N .
$$

In this paper, the distribution $G(r)$ is assumed to be known, either through the historical data of similar products or from customer investigation.

3.2. Imperfect and Periodic PM Strategy Model. A PM action generally corresponds to a series of repair activities, including systematic cleaning, inspection, lubricating, and renewing components. [10, 25]. 2An imperfect PM does not repair the failed item to a good-as-new state; instead, it repairs the failed item to a state of better-than-now. In order to measure the maintenance level of imperfect PM, experts and scholars usually model it with two approaches: virtual life decline and failure rate reduction $[26,27]$. As is well known, the periodic PM strategy is always implemented on the basis of a predetermined PM scheme, with which age decline could occur between the actual age and virtual age. This study uses virtual life reduction to model the maintenance degree of PM, and applies the model framework used by Kim et al. [28] to illustrate the age decline effort of imperfect PM strategy.

Assume that a set of PM activities of a product are planned at actual age $\tau_{1}, \tau_{2}, \ldots, \tau_{j}$. The effort of PM is supposed to result in the restoration of the product, so as to decline the system's virtual age significantly. This paper assumes that the damage accumulated during the time between the $j-1^{\text {th }}$ and the $j^{\text {th }}$ PM tasks only can be compensated by the $j^{\text {th }} \mathrm{PM}$ effectively, which results in an arithmetic decline of virtual age. The relationship between the effort of PM and the age reduction of the product can be 
TABLE 1: Mathematical notations and description.

\begin{tabular}{|c|c|}
\hline Notation & Description \\
\hline$W^{0}, U^{0}$ & Basic warranty's covered length and usage \\
\hline$W^{1}, U^{1}$ & Extended warranty's covered length and usage \\
\hline$R$ & Usage rate (random variables) \\
\hline$t, u$ & Product age and total usage, respectively \\
\hline$\lambda_{0}(x \mid r)$ & Conditional failure intensity of the product with no PM actions \\
\hline$N$ & Number of usage of rare scenarios \\
\hline$G(r), g(r)$ & Cumulative distribution function $(\mathrm{CDF})$ and probability density function (PDF) of the customer's usage rate \\
\hline$r_{\min }, r_{\max }$ & The minimum and maximum usage rates of the product \\
\hline$\left[r_{i-1}, r_{i}\right)$ & The $i^{\text {th }}$ usage rate interval, $i=1, \ldots, N$ \\
\hline$P_{r i}$ & The probability of usage rate $r$ belonging to the $i^{\text {th }}$ usage rate scenarios \\
\hline$m$ & PM level $[0 \leq m \leq M] . M=0$ corresponds to no PM and $M$ is the prespecified upper PM level, which is a constant \\
\hline$\delta(m)$ & The age reduction factor of PM with level $m[0 \leq m \leq M]$ \\
\hline$\tau_{j}$ & Actual age of the $j^{\text {th }} \mathrm{PM}$ actions scheduled, with $\tau_{0}=0$ \\
\hline$v_{j}$ & Virtual age of the product following the $j^{\text {th }} \mathrm{PM}$ action \\
\hline$T_{i}^{B}, m_{i}^{B}$ & Time interval and maintenance degree of PM during the BW period for the $i^{\text {th }}$ usage rate interval \\
\hline$T_{i}^{E}, m_{i}^{E}$ & Time interval and maintenance degree of PM during the EW period for the $i^{\text {th }}$ usage rate interval \\
\hline$n_{i}^{B}, n_{i}^{E}$ & The expected number of PM scheduled in the BW and EW or customers with the $i^{\text {th }}$ usage rate scenarios \\
\hline$W_{i}^{B}, W_{i}^{E}$ & Actual age limit of the BW and EW for customers with the $i^{\text {th }}$ usage rate scenarios \\
\hline$C_{\min }$ & Expected cost of each minimal repair \\
\hline$C_{\mathrm{PM}}(m)$ & Expected cost of each imperfect PM with maintenance level $m$ \\
\hline$E B_{\mathrm{PM}}^{r}, E B_{\mathrm{C}}^{r}$ & The expected PM cost and minimal repair cost for product with usage rate $r$ during the BW \\
\hline$E E_{\mathrm{PM}}^{r}, E E_{C}^{r}$ & The expected PM cost and minimal repair cost for product with usage rate $r$ during the EW \\
\hline$E_{i}^{B}, E_{i}^{E}$ & The expected total warranty cost for customers belonging to the $i^{\text {th }}$ usage rate scenarios \\
\hline$P_{i}^{E} \quad l$ & The purchasing ratio of PM contract for customers belonging to the $i^{\text {th }}$ usage rate scenarios \\
\hline
\end{tabular}

characterized by the age reduction factor $\delta(m)$, Where $m$ is the maintenance level of PM task. Then, $v_{j}$ right after implementing the $j^{\text {th }}$ PM task is delivered by

$$
v_{j}=v_{j-1}+\delta(m)\left(\tau_{j}-\tau_{j-1}\right), \quad m=1,2, \ldots, M .
$$

In this study, a large value of $m$ means greater PM effect; therefore, $\delta(m)$ is a decreasing function of $m$ with $\delta(0)=1$ and $\delta(M)=0$. The level of PM effect $m$ is supposed to remain unchanged throughout the BW or EW period, which is usually used in the existing PM models. This paper defines the functional relationship between $m$ and $\delta(m)$ by an exponentially decreasing function, $\delta(m)=(1+m) e^{-m}$. By iterative calculation, it can be obtained

$$
v_{j}=v_{0}+\delta(m)\left(\tau_{j}-\tau_{0}\right)
$$

This formula will be applied to explore the expected number of failures when a periodic and imperfect PM strategy is adopted in the next section.

\section{Mathematical Model of Two-Dimensional Warranty Cost}

In this section, a mathematical optimization model for a product sold with two-dimensional warranty is obtained to minimize the whole expected warranty cost of BW and EW from the manufacturer's view. For this purpose, the first section estimates the expected costs of PM and CM for the product in the BW and EW period, respectively. In the next section, considering the impact of the purchasing ratio of
EW contract $P_{i}^{E}$, the optimization model based on bi-level programming is presented.

4.1. Warranty Cost Model of BW and EW. Product warranty costs mainly include four different categories: PM cost in BW period, CM cost in BW period, $\mathrm{PM}$ cost in EW period, and $\mathrm{CM}$ cost in EW period. As mentioned above, the time interval and maintenance degree of PM can be different between BW and EW but must be consistent during the same warranty period. Generally speaking, the usage rate of a customer has an important impact on the degradation process of a product, with regard to the optimal maintenance strategy for the product during the BW or EW period. As shown in Figure 1, the warranty period this study assumes often varies with the usage rates of customers. This study has classified the customers based on their usage rates and then provided them with customized PM strategies, which are chartered by different schedule time intervals and maintenance degree.

Suppose $\left(W^{0}, U^{0}\right)$ and $\left(W^{1}, U^{1}\right)$ are the age and usage limit of the two-dimensional BW and EW period, respectively. Taking the $i^{\text {th }}$ usage rate scenarios as an example, $T_{i}^{B}, m_{i}^{B}$ represent the time interval and maintenance level of $\mathrm{PM}$ in the $\mathrm{BW}$ period, respectively; $T_{i}^{E}, m_{i}^{E}$ represent the schedule time interval and maintenance level of PM in the EW period, respectively; and $n_{i}^{B}, n_{i}^{E}$ are the number of PM in the BW and EW periods, respectively. $P_{i}^{E}$ is the purchasing ratio of $\mathrm{EW}$ contracts for customers belonging to the $i^{\text {th }}$ usage rate scenarios; $C_{\mathrm{PM}}(m)$ represents the expected PM costs of maintenance level $m$; $C_{\text {min }}$ is the expected cost of each minimal repair, conditional on usage rate $R \in\left[r_{i-1}, r_{i}\right)$; and the actual age limits of the BW and EW periods are: 


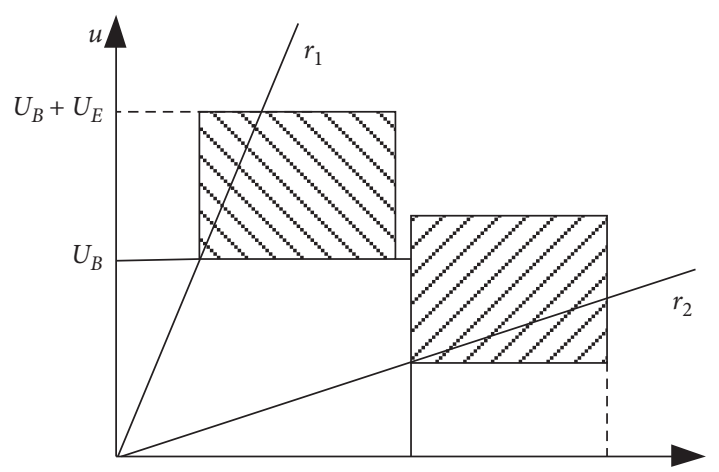

Figure 1: Two-dimensional warranty period.

$$
\begin{aligned}
& W_{i}^{B}=\min \left(W_{0}, \frac{U_{0}}{r_{i}}\right)= \begin{cases}W_{0}, & \text { if } r_{i} \leq \frac{U_{0}}{W_{0}}, \\
\frac{U_{0}}{r_{i}}, & \text { if } r_{i}>\frac{U_{0}}{W_{0}},\end{cases} \\
& W_{i}^{E}=\min \left(W_{1}, \frac{U_{1}}{r_{i}}\right)= \begin{cases}W_{1}, & \text { if } r_{i} \leq \frac{U_{1}}{W_{1}}, \\
\frac{U_{1}}{r_{i}}, & \text { if } r_{i}>\frac{U_{1}}{W_{1}} .\end{cases}
\end{aligned}
$$

Then, the number of PM carried out during the BW and EW for the $i^{\text {th }}$ usage rate scenario is given by

$$
\begin{aligned}
& n_{i}^{B}=\left[\frac{W_{i}^{B}}{T_{i}^{B}}\right], \\
& n_{i}^{E}=\left[\frac{W_{i}^{E}}{T_{i}^{E}}\right] .
\end{aligned}
$$

For the $i^{\text {th }}$ usage rate scenario, the values of these four types of warranty cost $E B_{\mathrm{PM}}^{r}\left(T_{i}^{B}, m_{i}^{B}\right), E B_{C}^{r}\left(T_{i}^{B}, m_{i}^{B}\right)$, $E E_{\mathrm{PM}}^{r}\left(T_{i}^{B}, m_{i}^{B}, T_{i}^{E}, m_{i}^{E}\right)$, and $E E_{C}^{r}\left(T_{i}^{B}, m_{i}^{B}, T_{i}^{E}, m_{i}^{E}\right)$ are as follows:

(1) The expected PM cost in the BW period is

$$
E B_{\mathrm{PM}}^{r}\left(T_{i}^{B}, m_{i}^{B}\right)=n_{i}^{B} * C_{\mathrm{PM}}\left(m_{i}^{B}\right) \text {. }
$$

(2) The expected CM cost in the BW period is

$$
E B_{C}^{r}\left(T_{i}^{B}, m_{i}^{B}\right)=C_{\min } *\left(\sum_{j=0}^{n_{i}^{B}-1} \int_{j * \delta\left(m_{i}^{B}\right) * T_{i}^{B}}^{j * \delta\left(m_{i}^{B}\right) * T_{i}^{B}+T_{i}^{B}} \lambda_{0}(t \mid r) \mathrm{d} t+\int_{\delta\left(m_{i}^{B}\right) * n_{i}^{B} * T_{i}^{B}}^{\delta\left(m_{i}^{B}\right) * n_{i}^{B} * T_{i}^{B}+W_{r}^{B}-n_{i}^{B} * T_{i}^{B}} \lambda_{0}(t \mid r) \mathrm{d} t\right)
$$

(3) Set $\tau_{i}^{E}=\delta\left(m_{i}^{B}\right) * n_{i}^{B} * T_{i}^{B}+W_{r}^{B}-n_{i}^{B} * T_{i}^{B}$ as the virtual life of products whose usage rate is $R \in\left[r_{i-1}, r_{i}\right]$ when they enter the EW period; thus, the expected PM cost in the EW period is

$$
E E_{\mathrm{PM}}^{r}\left(T_{i}^{B}, m_{i}^{B}, T_{i}^{E}, m_{i}^{E}\right)=n_{i}^{E} * C_{\mathrm{PM}}\left(m_{i}^{E}\right) .
$$

(4) The expected CM cost in the EW period is

$$
E E_{C}^{r}\left(T_{i}^{B}, m_{i}^{B}, T_{i}^{E}, m_{i}^{E}\right)=C_{\min } *\left(\sum_{j=0}^{n_{i}^{E}-1} \int_{j * \delta\left(m_{i}^{E}\right) * T_{i}^{E}+\tau_{i}^{E}}^{j * \delta\left(m_{i}^{E}\right) * T_{i}^{E}+T_{i}^{E}+\tau_{i}^{E}} \lambda_{0}(t \mid r) \mathrm{d} t+\int_{\delta\left(m_{i}^{E}\right) * n_{i}^{E} * T_{i}^{E}+\tau_{i}^{E}}^{\delta\left(m_{i}^{E}\right) * n_{i}^{E} * T_{i}^{E}+W_{r}^{E}-n_{i}^{E} * T_{i}^{E}+\tau_{i}^{E}} \lambda_{0}(t \mid r) \mathrm{d} t\right)
$$

Based on these four types of warranty cost, the total expected cost for the $i^{\text {th }}$ usage rate scenario can be calculated by

$$
E_{i}^{B}\left(T_{i}^{B}, m_{i}^{B}\right)+P_{i}^{E} * E_{i}^{E}\left(T_{i}^{B}, m_{i}^{B}, T_{i}^{E}, m_{i}^{E}\right)=\int_{r_{i-1}}^{r_{i}}\left(E B_{\mathrm{PM}}^{r}+E E_{\mathrm{PM}}^{r}+P_{i}^{E} *\left(E E_{\mathrm{PM}}^{r}+E E_{C}^{r}\right)\right) *\left(\frac{g(r)}{P_{r i}}\right) \mathrm{d} r
$$

4.2. Warranty Cost Optimal Model Based on Bi-Level Programming. As mentioned above, the maintenance strategy analysis for a product during BW or EW are mostly studied independently (IND) or are totally combined to be a unified warranty study (UND) as modeled by $\mathrm{Wu}$ [22] and Wang et al. [2]. However, in actual life, the purchasing ratio 
of the EW contract $P_{i}^{E}$ is less than 1, meaning that not everyone will purchase EW contract after BW ceases. Both IND and UND methods are unreal and cannot deliver the optimal PM strategy within the total warranty period.

It is well known that the maintenance strategy of the BW and the purchasing ratio of EW contracts will influence the optimal maintenance strategy for products during the EW period; in turn, the optimal maintenance strategy for products during the EW period will affect the maintenance strategy during the BW period. Referring to $[29,30]$, the bilevel programming can solve exactly the mutual influence between BW and EW. Therefore, this study applies the bilevel programming method to combine the maintenance strategies between BW and EW tightly, with the aim of minimizing the warranty costs as a whole.

In this study, based on the purchasing ratio of the EW contract for different usage rate scenarios $P_{i}^{E}$, the optimal and imperfect PM strategy of combined BW and EW by bi-level programming method (COD) is proposed, which is different from IND and UND models, as shown in Figure 2.

In the upper-level programming, the decision variables are $\left(T_{i}^{B}, m_{i}^{B}\right), i=1, \ldots, N$, and the aim is to minimize the overall expected warranty cost for all customers in the $\mathrm{BW}$ and EW periods. In the lower-level programming, the decision variables are $\left(T_{i}^{E}, m_{i}^{E}\right), i=1, \ldots, N$, and the aim is to minimize the expected warranty cost in the EW period for customers who purchase the EW contract, on condition that the value of $\left(T_{i}^{B}, m_{i}^{B}\right), i=1, \ldots, N$ is known. As the upperlevel decision variables, the maintenance strategies in the BW period determine the reliability of products when they enter the EW period. Then, they have a very important influence on the maintenance strategies in the EW period, which are the critical variables of the lower level. The objective function of the lower-level is a part of the objective function of the upper-level programming. Therefore, this model is a typical bi-level programming model, and the detailed model structure is as follows:

$$
\begin{aligned}
& \text { upper level: } \min _{T_{i}^{B}, m_{i}^{B}} E_{T}\left(T_{i}^{B}, m_{i}^{B}, T_{i}^{E}, m_{i}^{E}\right)=\sum_{i=1}^{N} P_{r i} *\left(E_{i}^{B}\left(T_{i}^{B}, m_{i}^{B}\right)+P_{i}^{E} * E_{i}^{E}\left(T_{i}^{B}, m_{i}^{B}, T_{i}^{E}, m_{i}^{E}\right)\right) \text {, } \\
& \text { s.t. } 0<T_{i}^{B} \leq W_{0}, \quad i=1, \ldots, N \text {, } \\
& 0 \leq m_{i}^{B} \leq M, \quad i=1,2, \ldots, N, \\
& \text { lower level: } \min _{T_{i}^{E}, m_{i}^{E}} E_{E}\left(T_{i}^{B}, m_{i}^{B}, T_{i}^{E}, m_{i}^{E}\right) \text {, } \\
& \text { s.t. } 0<T_{i}^{E} \leq W_{1}, \quad i=1, \ldots, N \text {, } \\
& 0 \leq m_{i}^{E} \leq M, \quad i=1,2, \ldots, N \text {, } \\
& \tau_{i}^{E}=\delta\left(m_{i}^{B}\right) * n_{i}^{B} * T_{i}^{B}+W_{r}^{B}-n_{i}^{B} * T_{i}^{B}, \quad i=1,2, \ldots, N .
\end{aligned}
$$

In this model, when the purchasing ratio of the EW contract $P_{i}^{E}, \forall i \in[1: N]$ is set to be 0 , the bi-level programming model will turn into two independent optimal models, of which the PM strategies for the BW and EW are studied independently (IND). On the contrary, when the purchasing ratio of EW contract $P_{i}^{E}, \forall i \in[1: N]$ is set to be 1 , it implies that all consumers will purchase EW services before the end of the BW period, and then the optimal maintenance researches for BW and EW can be modeled by a unified warranty period (UND), as studied by Wang et al. [2]. Therefore, the model proposed by this paper also can be perfectly applied to both IND and UND.

Generally speaking, if the values of $T_{i}^{B}, T_{i}^{E}$ are continuous variables in this model, the resolving process for this bilevel programming model will be an NP-hard problem, and it is quite hard to obtain the precise analytic solution. However, in fact, the time schedule and maintenance degree of PM are always treated as discrete variables for easily execution in practice. Therefore, the values of $T_{i}^{B}, T_{i}^{E}$ are treated as discrete variables. Then, the optimal time schedule $T_{i}^{B}, T_{i}^{E}$ and the corresponding maintenance degree $m_{i}^{B}, m_{i}^{E}$ are obtained by the global traversal method, which will be illustrated in more detail in the numerical analysis.

\section{Numerical Analysis}

As highlighted in the introduction, automobiles are one of the commonest industries for which two-dimensional warranties are applied successfully, so this paper uses a numerical example from the automotive industry to test and verify the effectiveness of the model. Firstly, by comparing the expected warranty costs of BW and EW for the different third optimal methods, IND, UND, and the method proposed by this paper, the model validity has been proven in Section 5.1. Secondly, through the warranty cost comparison of the unitary PM policy and customized PM policy proposed by this paper, the necessity of implementing customized PM strategies during the warranty period has been proven in Section 5.2. Finally, the sensitivity analysis of the main parameters, such as the purchasing ratio $P_{i}^{E}$ and the expected cost of minimal repair $C_{\min }$ are shown in Section 5.3 .

Referring to $[2,16]$, the main parameters are set as follows. Assume that an automobile component covered by a two-dimensional FRW policy is the product under consideration in this study. The BW period is 3 years and 60 thousand $\mathrm{km}$, that is $\left[W_{0}, U_{0}\right]=[3,6]$. Three EW regions 

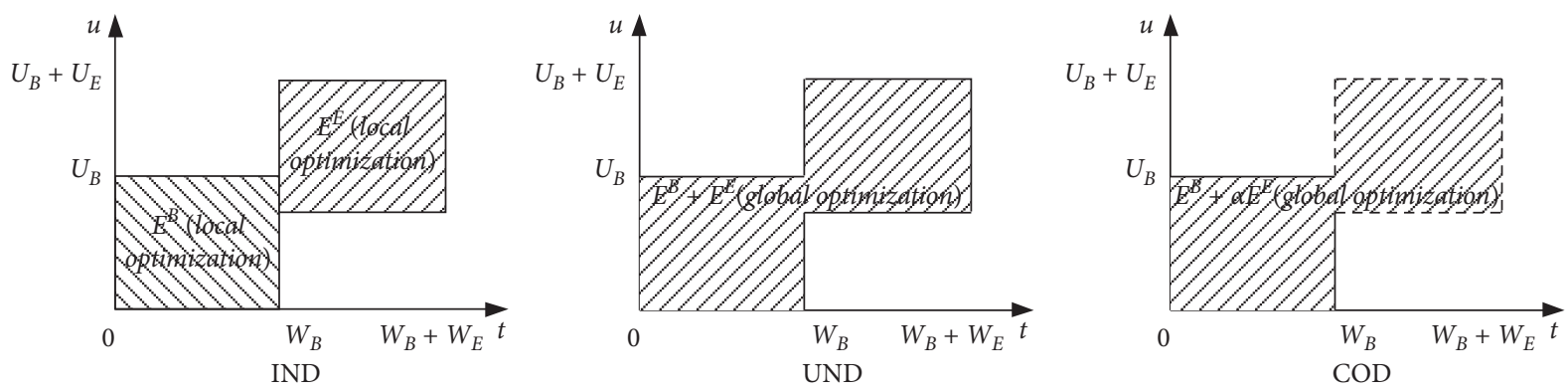

Figure 2: Comparison of IND, UND, and COD models.

are considered, $[3,3],[3,6],[6,6]$, respectively. The polynomial intensity function of the product failure given by (1) is used and taken to be

$$
\lambda_{0}(t \mid r)=\theta_{0}+\theta_{1} r+\left(\theta_{2}+\theta_{3} r\right) t=0.1+0.2 r+(0.7+0.7 r) t
$$

with the parameter values $\theta_{0}=0.1, \theta_{1}=0.2$, $\theta_{2}=0.7, \theta_{3}=0.7$.

Besides, $R$ is supposed to be uniformly distributed over $[0.5,3.5]$, the value range of which is then equally divided into 4 usage rate scenarios as $[0.5,1.25],[1.25,2]$, $[2,2.75],[2.75,3.5]$. What should be noted is that the distribution patterns of customer product usage rate have no or little impact on the study of optimal customized PM strategy proposed by this paper, and uniform distribution has been extensively applied to model the product usage rate, such as $[2,16,17]$.

Moreover, this study considers $\delta(m)=(1+m) e^{-m}$ for $m=0,1, \ldots, 5$, and the corresponding expected PM cost is $C_{\mathrm{PM}}(0)=0 \quad C_{\mathrm{PM}}(1)=10 \quad C_{\mathrm{PM}}(2)=30 \quad C_{\mathrm{PM}}(3)=60$, $C_{\mathrm{PM}}(4)=100$, and $C_{\mathrm{PM}}(5)=160$. The expected minimal repair cost is $C_{\min }=250$, which is consistent with that of [28], and the purchasing ratio of the EW contract is $P^{E}=$ $\left[\begin{array}{llll}0.4 & 0.5 & 0.6 & 0.6\end{array}\right]$ correspondingly.

It is noteworthy that time intervals and maintenance degree of periodic preventive maintenance are usually discrete in practice; however, most of the researches rarely consider this question by giving an oversimplified assumption of continuity. For the purpose of efficient maintenance management, this paper searches for the best, not necessarily optimal, maintenance degree and time interval over a coarse grid with $m$ and $t$ varying within $(0,5]$ and $\left(0, W^{0}\right]$. The expected costs of different maintenance degrees and time intervals in BW and EW are calculated by the method of global traversal, and the optimal maintenance interval and maintenance degree are obtained as described below, of which the basic unit of time schedule for PM is one month.

5.1. Model Validity Analysis. In this section, no matter what kind of decision scheme is to be adopted, the customized PM strategy will always be provided for customers according to their usage rate scenarios. Through numerical analysis, the optimal PM strategies under IND, COD, and UND are obtained, and the expected warranty costs for different usage rate scenarios during $\mathrm{BW}, \mathrm{EW}$, and both $\mathrm{BW}$ and $\mathrm{EW}$ are all calculated simultaneously, as presented in Tables 2 and 3.

The meanings of symbols in Tables 2 and 3 are all listed after Table 3. By comparing the optimal PM strategies and expected warranty cost of these three kinds of decision schemes for different EW regions (as shown in Figure 3), the following main findings can be obtained:

(1) By comparing the expected warranty cost of these three kinds of decision schemes for different warranty periods, it can be concluded that, for products covered by two-dimensional BW and EW, the maintenance strategies of BW and EW combining with bi-level programming model (COD) can effectively reduce the totally expected warranty cost, when compared to IND and UND, which is of great significance to the profit of the manufacturer.

(2) By comparing the optimal PM strategies for BW and EW under three kinds of decision schemes, it can be found that no matter what kind of EW regions are adopted, the optimal PM strategies for EW under different decision schemes always stay consistent, while the PM strategies for BW are changed obviously. Then, it can be summarized that the maintenance strategies for BW are the variables that can be adjusted to minimize the expected warranty cost for the whole warranty period. Therefore, it is worthwhile to consider the EW contract when taking decisions about the maintenance strategies for products during the BW period.

(3) By comparing the expected warranty cost of BW and EW under IND, UND, and COD, it can be found that when the purchasing ratio of EW is less than 1 , the warranty cost of EW under COD is less than that under IND but more than that under UND, while the warranty cost of $\mathrm{BW}$ under COD is more than that under IND but less than that under UND. On the whole, the expected cost of the whole warranty period under COD is minimal among these decision schemes, and the degree of reduction depends on the parameters such as product failure intensity, the purchasing ratio of EW, and so on.

(4) By comparing the optimal PM strategies between different usage ratio scenarios, it can be concluded that with the usage rate increasing, the schedule time interval of PM strategies will become shorter while 
TABLE 2: Preventive maintenance strategy and warranty cost of IND and COD.

\begin{tabular}{|c|c|c|c|c|c|c|c|c|c|c|c|}
\hline \multirow{2}{*}{$W$} & \multirow[b]{2}{*}{$Z$} & \multicolumn{5}{|c|}{ IND } & \multicolumn{5}{|c|}{ COD } \\
\hline & & $T^{B}$ & $M^{B}$ & $T^{E}$ & $M^{E}$ & $C_{I}\left(10^{\wedge} 3\right)$ & $T^{B}$ & $M^{B}$ & $T^{E}$ & $M^{E}$ & $C_{C}\left(10^{\wedge} 3\right)$ \\
\hline \multirow{5}{*}{3,3} & 1 & 10 & 3 & 10 & 3 & 1.4656 & 10 & 4 & 10 & 3 & 1.3663 \\
\hline & 2 & 10 & 4 & 7 & 3 & 1.6535 & 10 & 4 & 7 & 3 & 1.6535 \\
\hline & 3 & 7 & 3 & 7 & 3 & 1.484 & 7 & 4 & 7 & 3 & 1.4004 \\
\hline & 4 & 7 & 3 & 6 & 2 & 1.2765 & 7 & 4 & 6 & 2 & 1.212 \\
\hline & & $C_{B}$ & 4.266 & $C_{E}$ & 1.613 & 5.8797 & $C_{B}$ & 4.28 & $C_{E}$ & 1.353 & 5.6322 \\
\hline \multirow{5}{*}{3,6} & 1 & 10 & 3 & 10 & 3 & 1.6169 & 10 & 4 & 10 & 3 & 1.4922 \\
\hline & 2 & 10 & 4 & 10 & 4 & 2.1317 & 13 & 5 & 10 & 4 & 2.1237 \\
\hline & 3 & 7 & 3 & 7 & 3 & 2.0305 & 9 & 5 & 7 & 3 & 1.8447 \\
\hline & 4 & 7 & 3 & 7 & 3 & 1.7341 & 7 & 4 & 7 & 3 & 1.6011 \\
\hline & & $C_{B}$ & 4.266 & $C_{E}$ & 3.247 & 7.5131 & $C_{B}$ & 4.45 & $C_{E}$ & 2.609 & 7.0617 \\
\hline \multirow{5}{*}{6,6} & 1 & 10 & 3 & 10 & 3 & 2.0646 & 13 & 5 & 10 & 4 & 1.8426 \\
\hline & 2 & 10 & 4 & 10 & 4 & 2.1317 & 13 & 5 & 10 & 4 & 2.1237 \\
\hline & 3 & 7 & 3 & 7 & 3 & 2.0305 & 9 & 5 & 7 & 3 & 1.8447 \\
\hline & 4 & 7 & 3 & 7 & 3 & 1.7341 & 7 & 4 & 7 & 3 & 1.6011 \\
\hline & & $C_{B}$ & 4.266 & $C_{E}$ & 3.695 & 7.9609 & $C_{B}$ & 4.53 & $C_{E}$ & 2.884 & 7.4121 \\
\hline
\end{tabular}

TABLE 3: Preventive maintenance strategy and warranty cost of UND and COD.

\begin{tabular}{cccccccccc}
\hline & \multicolumn{8}{c}{$\mathrm{UND}$} \\
& $Z$ & \multicolumn{1}{c}{$T^{B}$} & $M^{B}$ & $C_{B}\left(10^{\wedge} 3\right)$ & $T^{E}$ & $M^{E}$ & $C_{E}\left(10^{\wedge} 3\right)$ & $C_{U}\left(10^{\wedge} 3\right)$ & $C_{C}\left(10^{\wedge} 3\right)$ \\
\hline & 1 & 13 & 5 & 1.0648 & 10 & 3 & 0.3287 & 1.3935 & 1.3663 \\
3, & 2 & 13 & 5 & 1.3927 & 7 & 3 & 0.3058 & 1.6985 & 1.6535 \\
3 & 3 & 7 & 4 & 1.0663 & 7 & 3 & 0.3341 & 1.4004 & 1.4004 \\
& 4 & 7 & 4 & 0.9293 & 6 & 2 & 0.2827 & 1.2120 & 1.212 \\
& & & $C_{B}$ & 4.4531 & & $C_{E}$ & 1.2513 & $\mathbf{5 . 7 0 4 4}$ & $\mathbf{5 . 6 3 2 2}$ \\
\hline & 1 & 13 & 5 & 1.0648 & 10 & 3 & 0.4425 & 1.5073 & 1.4922 \\
3, & 2 & 13 & 5 & 1.3927 & 10 & 4 & 0.7311 & 2.1238 & 2.1237 \\
6 & 3 & 9 & 5 & 1.141 & 7 & 3 & 0.7037 & 1.8447 & 1.8447 \\
& 4 & 7 & 5 & 1.0132 & 7 & 3 & 0.6067 & 1.6199 & 1.6011 \\
& & & $C_{B}$ & 4.6117 & & $C_{E}$ & 2.4839 & 7.0956 & 7.0617 \\
\hline & 1 & 13 & 5 & 1.0648 & 10 & 4 & 0.7778 & 1.8426 & 1.8426 \\
6, & 2 & 13 & 5 & 1.3927 & 10 & 4 & 0.7310 & 2.1237 & 2.1237 \\
6 & 3 & 9 & 5 & 1.141 & 7 & 3 & 0.7037 & 1.8447 & 1.8447 \\
& 4 & 7 & 5 & 1.0132 & 7 & 3 & 0.6067 & 1.6199 & 1.6011 \\
& & & $C_{B}$ & 4.6117 & & $C_{E}$ & 2.8191 & 7.4308 & 7.4121 \\
\hline
\end{tabular}

$W$ : extended warranty; $Z$ : different usage rate scenarios; $C_{I}$ : expected cost for IND; $C_{C}$ : expected cost for COD; $C_{U}$ : expected cost for UND; $C_{B}$ : expected cost of BW; $C_{E}$ : expected cost of BW.

the maintenance levels remain unchanged or change a little, meaning that the manufacturer should carry out more PM actions to slow down the degradation process of products caused by the high product usage rate. A reasonable $\mathrm{PM}$ scheme is a trade-off between $\mathrm{PM}$ cost and CM cost.

5.2. Model Validity Analysis of Customized PM Strategy. As mentioned above, the customers are divided into four different types based on their usage rate, and customized PM strategies are provided for each of them. In order to test the effectiveness of these customized PM strategies, this study has compared the expected warranty cost between the customized PM strategies and uniform PM strategies during

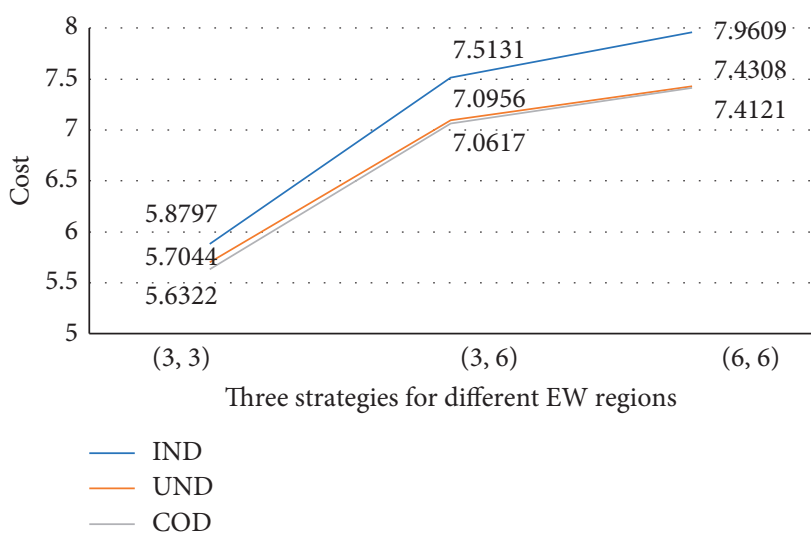

FIGURE 3: The expected warranty cost of different strategies.

both BW and EW periods; it is supposed that the bi-level programming model is also used to combine the maintenance strategies between BW and EW, and all the other parameters remain unchanged, then the optimal maintenance strategies and expected warranty cost of the uniform PM strategies are obtained, as shown in Table 4.

As shown above, for products sold with two-dimensional BW and different optional EW contracts, the optimal unitary PM strategies for each of them are obtained and the expected warranty costs for different usage rate scenarios are also calculated simultaneously. By comparing the expected warranty costs between unitary PM strategies and customized PM strategies for different usage rate scenarios, it can be found that the customized PM strategies for customers with different usage rates can lower warranty cost covered by the manufacturer availability, thus the total warranty cost will decrease obviously.

5.3. Sensitivity Analysis of Main Parameters. In this section, the sensitivity analysis of main parameters such as the purchasing ratio $P_{i}^{E}$ and the expected cost of minimal repair 
TABLE 4: Maintenance strategy and warranty cost of unitary PM and customized PM.

\begin{tabular}{|c|c|c|c|c|c|c|}
\hline$W$ & $\left(T^{B} M^{B} T^{E} M^{E}\right)$ & $Z$ & $C_{B}(10 \wedge 3)$ & $C_{E}(10 \wedge 3)$ & $C_{U}\left(10^{\wedge} 3\right)$ & $C_{C}(10 \wedge 3)$ \\
\hline \multirow{5}{*}{33} & \multirow{5}{*}{7462} & 1 & 1.0961 & 0.3992 & 1.4953 & 1.3663 \\
\hline & & 2 & 1.3645 & 0.3754 & 1.7399 & 1.6535 \\
\hline & & 3 & 1.0663 & 0.3464 & 1.4127 & 1.4004 \\
\hline & & 4 & 0.9293 & 0.2827 & 1.2120 & 1.212 \\
\hline & & TOTAL & 4.4562 & 1.4037 & 5.8599 & 5.6322 \\
\hline \multirow{5}{*}{36} & \multirow{5}{*}{7473} & 1 & 1.0961 & 0.5181 & 1.6142 & 1.4922 \\
\hline & & 2 & 1.3645 & 0.8617 & 2.2262 & 2.1237 \\
\hline & & 3 & 1.0663 & 0.7900 & 1.8563 & 1.8447 \\
\hline & & 4 & 0.9293 & 0.6718 & 1.6011 & 1.6011 \\
\hline & & TOTAL & 4.4562 & 2.8415 & 7.2977 & 7.0617 \\
\hline \multirow{5}{*}{66} & \multirow{5}{*}{7473} & 1 & 1.0961 & 0.9422 & 2.0383 & 1.8426 \\
\hline & & 2 & 1.3645 & 0.8617 & 2.2262 & 2.1237 \\
\hline & & 3 & 1.0663 & 0.7900 & 1.8563 & 1.8447 \\
\hline & & 4 & 0.9293 & 0.6718 & 1.6011 & 1.6011 \\
\hline & & TOTAL & 4.4562 & 3.2656 & 7.7218 & 7.4121 \\
\hline
\end{tabular}

TABle 5: Sensitivity analysis of purchasing ratio $P^{E}$.

\begin{tabular}{|c|c|c|c|c|c|c|c|c|c|c|c|c|c|c|c|c|}
\hline \multirow{2}{*}{$P^{E}$} & \multicolumn{4}{|c|}{1} & \multicolumn{4}{|c|}{2} & \multicolumn{4}{|c|}{3} & \multicolumn{4}{|c|}{4} \\
\hline & $T^{B}$ & $M^{B}$ & $T^{E}$ & $M^{E}$ & $T^{B}$ & $M^{B}$ & $T^{E}$ & $M^{E}$ & $T^{B}$ & $M^{B}$ & $T^{E}$ & $M^{E}$ & $T^{B}$ & $M^{B}$ & $T^{E}$ & $M^{E}$ \\
\hline 0.1 & 10 & 4 & 10 & 4 & 10 & 4 & 10 & 4 & 7 & 4 & 7 & 3 & 7 & 4 & 7 & 3 \\
\hline 0.3 & 10 & 4 & 10 & 4 & 10 & 4 & 10 & 4 & 7 & 4 & 7 & 3 & 7 & 4 & 7 & 3 \\
\hline 0.5 & 13 & 5 & 10 & 4 & 13 & 5 & 10 & 4 & 7 & 4 & 7 & 3 & 7 & 4 & 7 & 3 \\
\hline 0.7 & 13 & 5 & 10 & 4 & 13 & 5 & 10 & 4 & 8 & 5 & 7 & 3 & 7 & 4 & 7 & 3 \\
\hline 0.9 & 13 & 5 & 10 & 5 & 13 & 5 & 10 & 4 & 9 & 5 & 7 & 3 & 7 & 5 & 7 & 3 \\
\hline
\end{tabular}

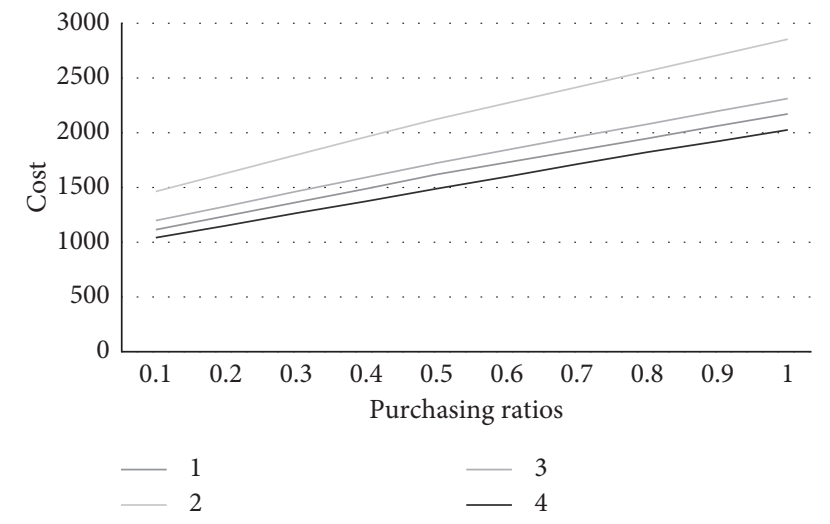

Figure 4: The expected warranty cost of different usage rate scenarios.

$C_{\text {min }}$ are discussed in detail. Generally speaking, the bi-level programming model proposed by this paper is mainly based on the purchasing ratio of EW contract $P_{i}^{E}$. For testing the effect of $P_{i}^{E}$ on the maintenance strategies of BW and EW, this study has calculated all the optimal PM strategies of the four different usage rate scenarios when their purchasing ratio changed from 0.1 to 0.9 , with the $\mathrm{EW}$ region of $[3,6]$, while all the other model parameters remain unchanged. The result is listed in Table 5 .

As shown in Table 5, with the purchasing ratio of the PM contract increasing, the schedule time of PM strategies for $\mathrm{BW}$ becomes longer or the maintenance level of PM becomes bigger. However, the optimal PM strategies for the EW period remain unchanged, and it can be summarized that the adjustment of maintenance strategies during the BW period can effectively reduce the total warranty cost when the value of $P_{i}^{E}$ is changed.

At the same time, this study has also compared the expected warranty cost between different usage rate scenarios for purchasing ratios ranging from 0.1 to 1 , with the purchasing ratio of $\mathrm{PM}$ contract for different usage rate scenarios staying the same, as is shown in Figure 4.

By comparing the expected warranty cost during the whole warranty period between different usage rate scenarios, it is obvious that the expected warranty cost does not follow up with the growth of the usage rate, and the expected warranty cost for the second usage rate scenarios is always the highest among all usage rate scenarios, for any circumstance. This phenomenon means that the expected warranty costs are not positively correlated with the product usage rate, and it may be caused by the fact that customers belonging to the second usage rate scenarios have a longer warranty period than the higher usage rate scenarios, while they have more expected product failures than the lower usage rate scenarios.

Except the purchasing ratio $P_{i}^{E}$, the expected cost of each minimal repair $C_{\min }$ or $\mathrm{PM}$ cost with different maintenance levels $C_{\mathrm{PM}}(m)$ are the main parameters impacting the optimal PM strategies during the whole warranty period. Or more precisely, the ratio of the expected cost of minimal repair to preventive maintenance for different levels is the main parameter affecting the optimal maintenance strategies. Suppose that the expected cost of PM with different maintenance level $m$ remains 
TABLe 6: Sensitivity analysis of the expected cost of minimal repair $C_{\min }$.

\begin{tabular}{cccccccccccccccccc}
\hline$C_{\min }$ & $T^{B}$ & $M^{B}$ & $T^{E}$ & $M^{E}$ & $T^{B}$ & $M^{B}$ & $T^{E}$ & $M^{E}$ & $T^{B}$ & $M^{B}$ & $T^{E}$ & $M^{E}$ & $T^{B}$ & $M^{B}$ & $T^{E}$ & $M^{E}$ \\
\hline 50 & 13 & 3 & 13 & 2 & 13 & 3 & 13 & 2 & 14 & 4 & 14 & 2 & 11 & 3 & 11 & 2 \\
100 & 13 & 4 & 13 & 3 & 13 & 4 & 13 & 3 & 14 & 4 & 9 & 3 & 11 & 4 & 11 & 3 \\
150 & 13 & 4 & 13 & 3 & 13 & 4 & 10 & 3 & 9 & 4 & 9 & 3 & 11 & 4 & 7 & 3 \\
200 & 13 & 4 & 10 & 3 & 13 & 5 & 10 & 4 & 9 & 4 & 7 & 3 & 7 & 4 & 7 & 3 \\
250 & 10 & 4 & 10 & 3 & 13 & 5 & 10 & 4 & 9 & 5 & 7 & 3 & 7 & 4 & 7 \\
300 & 10 & 4 & 10 & 4 & 10 & 5 & 8 & 4 & 9 & 5 & 7 & 4 & 7 & 5 & 7 \\
350 & 10 & 4 & 10 & 4 & 10 & 5 & 8 & 4 & 9 & 5 & 7 & 4 & 7 & 5 & 7 \\
400 & 10 & 5 & 8 & 4 & 10 & 5 & 8 & 4 & 7 & 5 & 7 & 4 & 7 & 5 & 7 \\
450 & 10 & 5 & 8 & 4 & 8 & 5 & 8 & 4 & 7 & 5 & 7 & 4 & 7 & 5 & 6 \\
500 & 10 & 5 & 8 & 4 & 8 & 5 & 8 & 4 & 7 & 5 & 7 & 4 & 7 & 5 & 6 \\
\hline
\end{tabular}

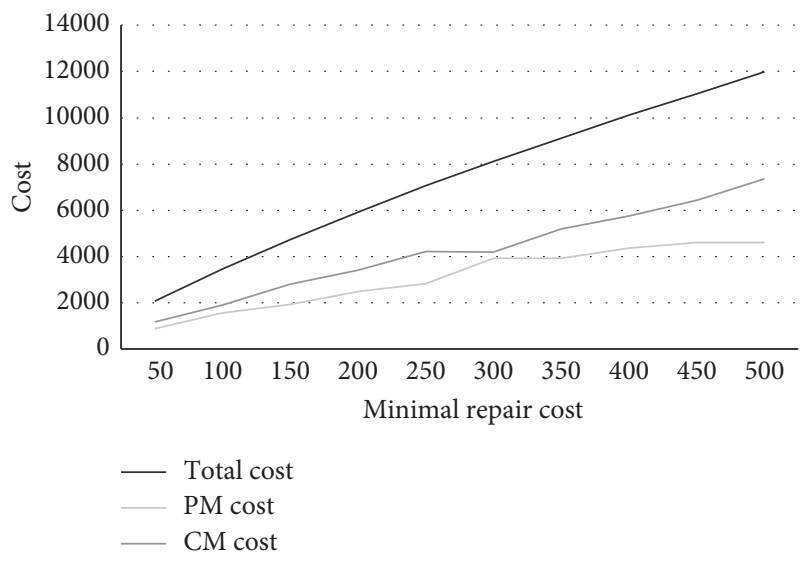

Figure 5: The expected cost for different $C_{\min }$.

unchanged, this paper has calculated the optimal PM strategies under all circumstances, when the expected cost of each minimal repair changes from 50 to 500 , adding 50 each time.

Table 6 shows that with the increase in the expected minimal repair cost, the schedule time interval of PM becomes shorter and the maintenance level of PM becomes bigger; this means that the manufacturer has to implement more PM actions to reduce the expected failure of products during the whole warranty period, for the sake of avoiding expensive maintenance costs.

What is more, this study has also compared the expected PM cost, the expected CM cost, and the total cost when the expected minimal repair cost changes from 50 to 500 , as shown in Figure 5.

It is obvious to find that all the expected warranty costs, such as PM cost, CM cost, and total cost, will increase when the expected minimal repair cost changes to bigger values, which is very easy to understand. However, what needs to be pointed out is that the expected PM cost is invariably less than the expected CM cost, which is very close when the minimal repair cost is 300 . The reason for this is that the optimal PM strategies for products during the whole warranty period are a balance of PM cost and CM cost, with the aim of minimizing the full warranty cost, which is always obtained when the cost of PM and $\mathrm{CM}$ is the same or thereabouts.

\section{Conclusions}

Two-dimensional warranty has played a significant role in improving product quality and competitiveness. Two-dimensional basic warranty (BW) and extended warranty (EW) are not incorporated in an integrated manner, which could potentially result in additional warranty costs for manufacturers. Due to the heterogeneity of customers' usage rates, the unitary PM strategy cannot meet the different demands of all the customers. In the manufacturer's view, it is absolutely essential to combine the BW and EW contracts, and consider the heterogeneity of customers' usage rates $[2,16]$. Since studies on two-dimensional warranties combining BW with EW and customized PM strategies are fairly limited, this paper provides a customized PM strategy combining BW with EW for products sold with two-dimensional warranty.

The main innovation of this study is twofold. First, this study applies the bi-level programming technique for combining the maintenance strategies between BW and EW effectively. Generally, the maintenance strategies of BW would influence the maintenance cost of EW. When the manufacturer makes the maintenance strategies within the BW period, the warranty cost of EW would be considered simultaneously in the bi-level programming model. Hence, the perfection of the BW strategies in the early stage would reduce the warranty cost within the EW period, thereby reducing the total warranty cost. Second, this study sets up an optimal customized PM strategy model by considering the heterogeneity of customers' usage rates. Manufacturers can make optimal PM strategies based on the customers' usage rates, which would contribute to improve the satisfaction of customers with different usage rates. Compared with IND and UND, the proposed model can significantly decline the warranty cost covered by the manufacturer during the whole warranty period. Therefore, this paper establishes a perfect research framework for the optimization of the maintenance strategy during the whole warranty period, which can provide scientific guidance for manufacturers to optimize the PM strategy during the BW and EW periods, thereby helping manufacturers to form more reasonable PM strategies for different customers.

PM strategies during the $\mathrm{BW}$ and $\mathrm{EW}$ period have been optimized in this paper. In practice, the EW period often 
varies for customers with different usage rates. Therefore, it is worth studying the PM strategies and the design of the EW period for customers with different usage rates as a whole under the constraint of maintenance cost, thereby providing a customized maintenance strategy based on the situation of the customers' usage rates in the future.

In addition, the interactions among the cost of EW, the purchasing ratio of the two-dimensional warranty service, and the optimization of predictive maintenance strategies are not taken into account in this paper. Since the objective of manufacturers is to maximize profits, the optimal maintenance strategies within the whole warranty period are required by considering two-dimensional extended warranty price and this is also an interesting topic for future research.

Last but not least, the expected cost of minimal maintenance and PM are supposed to be constant during the whole warranty in this paper. However, in practice, the cost of corrective repair and PM could add to the product's age or usage; thus, it is meaningful to explore the cost of minimal repair or PM as a function of the age and usage of the product when making decisions on maintenance strategies during the warranty period.

\section{Data Availability}

The warranty data used to support the findings of this study are included within the article.

\section{Conflicts of Interest}

The authors declare that they have no conflicts of interest.

\section{Acknowledgments}

This research was partially funded by the National Natural Science Foundation of China (Grant Nos. 71532008 and 71701200), China Postdoctoral Science Foundation (Grant No. 2016M590525), and Jiangsu Postdoctoral Science Foundation, China (Grant No. 1601246C).

\section{References}

[1] D. N. P. Murthy and I. Djamaludin, "New product warranty: a literature review," International Journal of Production Economics, vol. 79, no. 3, pp. 231-260, 2002.

[2] Y. Wang, Z. Liu, and Y. Liu, "Optimal preventive maintenance strategy for repairable items under two-dimensional warranty," Reliability Engineering \& System Safety, vol. 142, no. 5, pp. 326-333, 2015.

[3] A. A. Taleizadeh and M. Mokhtarzadeh, "Pricing and twodimensional warranty policy of multi-products with online and offline channels using a value-at-risk approach," Computers \& Industrial Engineering, vol. 148, Article ID 106674, 2020.

[4] Q. Hu, Y. Bai, J. Zhao, and W. Cao, "Modeling spare parts demands forecast under two-dimensional preventive maintenance policy," Mathematical Problems in Engineering, vol. 2015, Article ID 728241, 9 pages, 2015.

[5] Z. H. Cheng, Z. Y. Yang, Z. Zhao, Y. B. Wang, and Z. W. Li, "Preventive maintenance strategy optimizing model under two-dimensional warranty policy," Eksploatacja I Niezawodnosc-Maintenance and Reliability, vol. 17, no. 3, pp. 365-372, 2015.

[6] Y. C. Han, Y. S. Bai, X. J. Wu, and X. Y. Li, "Extended warranty models," A Literature Review, Advanced Materials Research, vol. 1030-1032, pp. 2566-2572, 2014.

[7] M. Finkelstein and M. Shafiee, "Preventive maintenance for systems with repairable minor failures," Journal of Risk \& Reliability, vol. 231, no. 2, 2017.

[8] M. Park, "Determination of optimal warranty period with preventive maintenance actions for items from heterogeneous populations," Mathematical Problems in Engineering, vol. 2020, Article ID 3480959, 12 pages, 2020.

[9] Y.-S. Huang, W.-Y. Gau, and J.-W. Ho, "Cost analysis of twodimensional warranty for products with periodic preventive maintenance," Reliability Engineering \& System Safety, vol. 134, pp. 51-58, 2015.

[10] X. Wang and C. Su, "A two-dimensional preventive maintenance strategy for items sold with warranty," International Journal of Production Research, vol. 54, no. 19, pp. 1-15, 2016.

[11] S. Chukova, Y. Hayakawa, and M. R. Johnston, "Optimal twodimensional warranty repair strategy," Proceedings of the Institution of Mechanical Engineers, Part O: Journal of Risk and Reliability, vol. 221, no. 4, pp. 265-273, 2007.

[12] Z.-L. Lin, Y.-S. Huang, and C.-C. Fang, "Non-periodic preventive maintenance with reliability thresholds for complex repairable systems," Reliability Engineering \& System Safety, vol. 136, pp. 145-156, 2015.

[13] A. Nasrum, U. S. Pasaribu, and H. Husniah, "Service contract with periodic preventive maintenance for a dump truck sold with a two-dimensional warranty," AIP Conference Proceedings, vol. 1716, 2016.

[14] K. Shahanaghi, R. Noorossana, S. G. Jalali-Naini, and M. Heydari, "Failure modeling and optimizing preventive maintenance strategy during two-dimensional extended warranty contracts," Engineering Failure Analysis, vol. 28, no. 3, pp. 90-102, 2013.

[15] Y.-H. Chien, Z. G. Zhang, and X. Yin, "On optimal preventive-maintenance policy for generalized polya process repairable products under free-repair warranty," European Journal of Operational Research, vol. 279, no. 1, pp. 68-78, 2019.

[16] C. Su and X. Wang, "A two-stage preventive maintenance optimization model incorporating two-dimensional extended warranty," Reliability Engineering \& System Safety, vol. 155, no. 3, pp. 169-178, 2016.

[17] Y.-S. Huang, C.-D. Huang, and J.-W. Ho, "A customized twodimensional extended warranty with preventive maintenance," European Journal of Operational Research, vol. 257, no. 3, pp. 971-978, 2017.

[18] S. Bouguerra, N. Rezg, and C. Anis, "A decision model for adopting an extended two-dimensional warranty region under different maintenance policies," in Proceedings of the 4th International Conference On Industrial Engineering and Systems Management, pp. 1141-1151, Metz, France, May 2011.

[19] Z. Zhang, S. He, Z. He, and A. Dai, "Two-dimensional warranty period optimization considering the trade-off between warranty cost and boosted demand," Computers \& Industrial Engineering, vol. 130, pp. 575-585, 2019.

[20] M. Shafiee, S. Chukova, M. Saidi-Mehrabad, and S. T. Akhavan Niaki, "Two-dimensional warranty cost analysis for secondhand products," Communications in Statistics - Theory and Methods, vol. 40, no. 4, pp. 684-701, 2011. 
[21] N. Jack, B. P. Iskandar, and D. N. P. Murthy, "A repair-replace strategy based on usage rate for items sold with a two-dimensional warranty," Reliability Engineering \& System Safety, vol. 94, no. 2, pp. 611-617, 2009.

[22] S. Wu, "Construction of asymmetric copulas and its application in two-dimensional reliability modelling," European Journal of Operational Research, vol. 238, no. 2, pp. 476-485, 2014.

[23] D. N. Murthy, J. Baik, R. Wilson, and M. Bulmer, "Two-dimensional failure modeling," in Springer Handbook of Engineering Statistics, H. Pham, Ed., pp. 97-111, Springer, Dordrecht, Netherlands, 2006.

[24] C. Su and X. Wang, "Optimal upgrade policy for used products sold with two-dimensional warranty," Quality and Reliability Engineering International, vol. 32, no. 8, pp. 2889-2899, 2016.

[25] A. B. Mabrouk, A. Chelbi, and M. Radhoui, "Optimal imperfect preventive maintenance policy for equipment leased during successive periods," International Journal of Production Research, vol. 54, no. 17, pp. 1-16, 2016.

[26] L. Doyen and O. Gaudoin, "Classes of imperfect repair models based on reduction of failure intensity or virtual age," Reliability Engineering \& System Safety, vol. 84, no. 1, pp. 45-56, 2004.

[27] M. Kijima, H. Morimura, and Y. Suzuki, "Periodical replacement problem without assuming minimal repair," European Journal of Operational Research, vol. 37, no. 2, pp. 194-203, 1988.

[28] C. S. Kim, I. Djamaludin, and D. N. P. Murthy, "Warranty and discrete preventive maintenance," Reliability Engineering \& System Safety, vol. 84, no. 3, pp. 301-309, 2004.

[29] K. Lachhwani and A. Dwivedi, "Bi-level and multi-level programming problems: taxonomy of literature review and research issues," Archives of Computational Methods in Engineering, vol. 25, no. 4, pp. 847-877, 2018.

[30] R. Lotfi, N. Mardani, and G. W. Weber, "Robust bi-level programming for renewable energy location," International Journal of Energy Research, vol. 1, pp. 1-14, 2020. 